\title{
Transmission and reflection properties of two-dimensional finite metal crystals
}

\author{
Agata Roszkiewicz and Wojciech Nasalski ${ }^{\mathrm{a}}$ \\ Institute of Fundamental Technological Research, Polish Academy of Sciences, Adolfa Pawiñskiego 5b, 02-106 Warsaw, Poland
}

Received 12 November 2016

Published online 6 July 2017

(c) The Author(s) 2017. This article is published with open access at Springerlink.com

\begin{abstract}
Optical characteristics of a finite two-dimensional silver stripe photonic crystal of a square lattice are numerically analysed with use of multilayer Rigorous Coupled Wave Analysis. Qualitative changes in optical response of the crystal originated from modifications of the thickness and filling factors of each layer and the polarization direction of the incident wave are shown. The crystal manifests its various characteristics in wideband or narrowband reflection and transmission, while absorption remains low. The behaviour of the crystal is determined by its structure geometry yielding excitation of localized plasmons and collective modes together with interactions between them. The optical response of the square lattice structure is also compared with the response of a triangular lattice crystal.
\end{abstract}

\section{Introduction}

Subwavelength metal periodic gratings can strongly interact with electromagnetic (EM) field waves [1]. This interaction can be enhanced in the case of polaritonic crystals build out of many stacked grating layers [2]. The twodimensional (2D) crystal is composed of horizontally 1D periodic sets of dielectric stripes forming a second 1D periodic sequence of layers in the orthogonal to the horizontal plane vertical direction. The stripes in all layers are arranged in parallel and the metal gratings are buried in a dielectric host material. In general, metal photonic crystals offer additional possibilities in comparison with dielectric crystals in modelling their overall hybrid behaviour by using plasmonic properties of metals [2]. Plasmonic resonances may introduce new phenomena that can further modify the optical response of the structure. Such metal photonic crystals can sustain several phenomena of high importance in applied optics like polarization optical filtering [3], thermal emission [4], enhanced absorption [5], negative refraction [6] or omnidirectional reflection [7]. The main advantage of the stripe crystal structure is that it can be produced sequentially layer by layer with standard photolithography or electron beam lithography techniques $[1,2]$.

Here we analyse numerically the two-dimensional silver photonic stripe structure of a rectangular symmetry with use of multilayer Rigorous Coupled Wave Analysis. In the analysed configuration the stripes in each layer are placed exactly at the same horizontal position without any horizontal spatial shift. This kind of the structure can exhibit several optical properties dependent on its geomet-

\footnotetext{
${ }^{a}$ e-mail: wnasal@ippt.pan.pl
}

rical parameters. Significant changes in transmission and reflection field spectra can be obtained by changing the filling fraction, the thickness of the structure elements or the polarization direction of the incident wave. By properly choosing these parameters it is possible to design a broadband reflector, a broadband transmitter or a narrowband transmitter. We also compare the optical response of this type of the crystal with a similar one, where the additional horizontal spatial shift by half of the grating period is introduced in every second layer (see Fig. 1). It appears that, despite significant changes of the structure geometry, the optical response of the crystal with this horizontally symmetric shift between the layers does not differ qualitatively from this without a shift, for most configurations of the structure parameters.

\section{Geometry of the structure and its modelling}

The configuration analysed first is depicted in Figure 1a. Each layer of the crystal consists of a 1D rectangular silver grating of the same thickness $d$ and the filling factor $f$. In the crystal the metal stripes in the subsequent layers do not cover the whole area of the structure, what results in presence of vertical air areas in planes perpendicular to the propagation direction.

In an infinite photonic crystal only propagation modes can exist [8]. However, the semi-infinite in the vertical direction spatial structure of the crystal may support also evanescent and surface modes $[1,9]$. Here we analyse the semi-infinite structure of the $2 \mathrm{D}$ crystal, which consists of four layers resulting of four periods in the stacking direction. Note that increasing further the number of layers of the modelled crystal might lead to slight changes 
a)

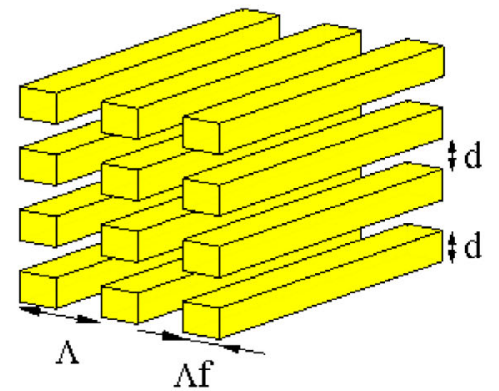

b)

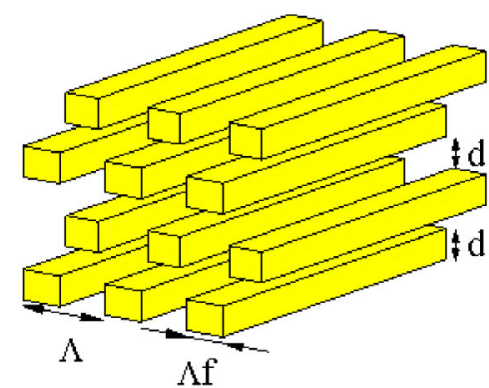

Fig. 1. Configuration of the problem. (a) Metal photonic crystal consists of 4 identical grating layers, each of thickness $d$, separated vertically by $d$. Width of the metal stripe in each layer is $\Lambda f$. Period in $x$ direction is equal to $\Lambda$. (b) Metal photonic crystal with a horizontal spatial shift by half of the grating period introduced in every second layer. The other geometrical parameters remain the same.
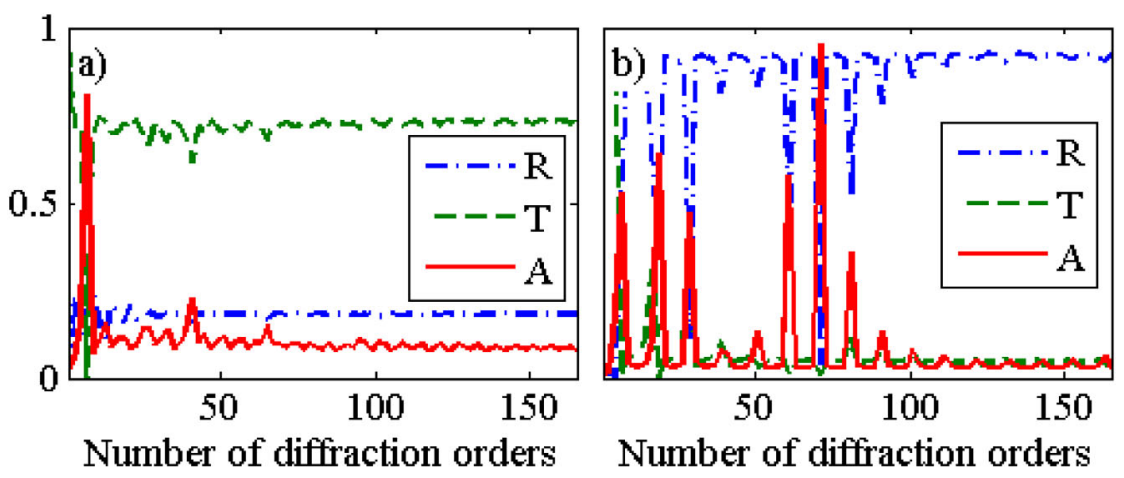

Fig. 2. Convergence of reflection $(R$ - blue dotted-dashed line), transmission $(T-$ green dashed line $)$ and absorption $(A-$ red solid line) for configuration with (a) rectangular lattice, $f=0.65, d=200 \mathrm{~nm}$ and $\lambda=700 \mathrm{~nm}$ and incidence of TM polarization, (b) triangular lattice, $f=0.2, d=200 \mathrm{~nm}$ and $\lambda=630 \mathrm{~nm}$ and incidence of TE polarization.

of the optical response of the structure, but still preserving qualitative characteristics of the structure behaviour. This issue will be addressed in Section 7.

A plane wave of linear polarization is incident normally onto a surface of the crystal from air $\left(\varepsilon_{1}=1\right)$. Polarization of the incident plane wave is defined with respect to the arrangement of the metal stripes. For transverse magnetic (TM) polarization the magnetic field vector is oriented parallel the metal stripes meanwhile for transverse electric (TE) polarization the electric field takes this direction. The optical response of the structure can be controlled by its material and geometry parameters. The most pronounced changes in the crystal's reflection, transmission and absorption efficiency are caused by changes of the filling factor $f$ (i.e. proportional to the width of the metal stripes) as well as of the thickness $d$ of each layer. Here we keep the horizontal period $\Lambda$ of the gratings constant and equal to $400 \mathrm{~nm}$. However, further optimization of the structure might require varying the following parameters: the separation between the subsequent layers (here equal to $d$ ) and the period $\Lambda$ of the structure in the horizontal direction $x$.

Numerical analysis of the optical response of the crystal was performed using the Rigorous Coupled Wave Analysis [10], with implementation of the scattering matrix algorithm [11] and the factorization rules [12]. There were
145 space Fourier components taken into account in the horizontal direction $x$. The wavelength dependence of dielectric function of silver was numerically fitted to the experimental data [13]. The wavelength range in the analysis is chosen to be within the range of low loss of silver and thereby the field-structure interactions could be analysed with use of Drude model as well [14]. Reflection or transmission coefficients are calculated as the ratio of the field components - of the real power carried by the reflected or transmitted wave normal to the boundary - to the corresponding component of the real power associated with the incident wave [15].

To show the correctness of our numerical algorithm, we present in Figure 2 the convergence of the analysis regarding the number of Fourier components taken into consideration for two cases: with rectangular lattice and TM incident polarization, $f=0.65, d=200 \mathrm{~nm}$ and $\lambda=700 \mathrm{~nm}$ (corresponding to the case presented in Fig. 4b) and triangular lattice, filling factor $f=0.2, d=200 \mathrm{~nm}$ and $\lambda=630 \mathrm{~nm}$ and incidence of TE polarization (corresponding to the case presented in Fig. 8b). Although good convergence is obtained even for 100 diffraction orders, we use 145 diffraction orders in order to perform calculations with minimal inaccuracy.

Here we assume that the metal crystal is embedded in the medium with dielectric constant equal to one 
TM

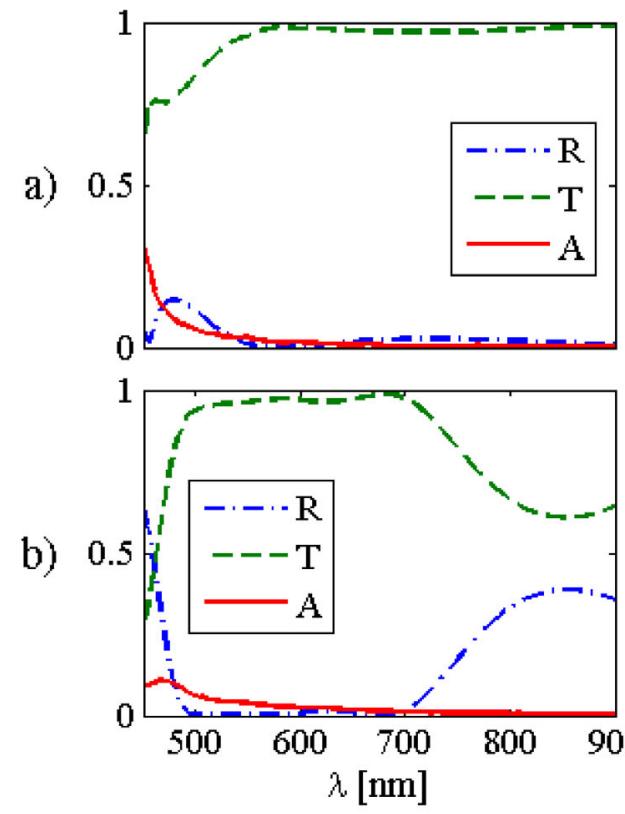

TE
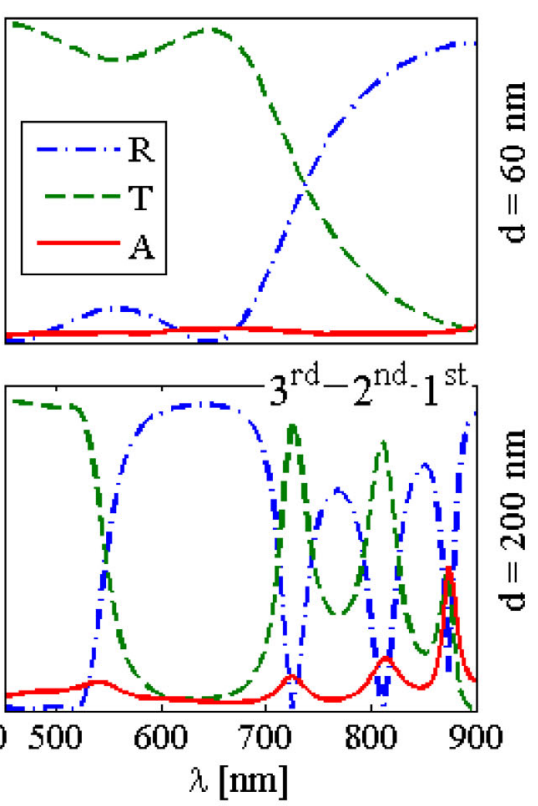

Fig. 3. Wavelength dependence of reflection $(R$ - blue dotted-dashed line), transmission ( $T$ - green dashed line) and absorption ( $A$ - red solid line) for the configurations with filling factor $f=0.2$. (a) The thickness $d=60 \mathrm{~nm}$ for incidence of TM (left) and TE (right) polarization; (b) the thickness $d=200 \mathrm{~nm}$ for incidence for TM (left) and TE (right) polarization. The resonances of the structure: 1 st order resonance at $\lambda=873 \mathrm{~nm}, 2 \mathrm{nd}$ order resonance at $\lambda=812 \mathrm{~nm}$ and $3 \mathrm{rd}$ order resonance at $\lambda=725 \mathrm{~nm}$ are also indicated.

(air is considered as the host medium). However, the RCWA method allows for scaling of the parameters with respect to dielectric constant value of the host medium. This means that the presented results are also valid for media with dielectric constant higher than one, together with dielectric permittivity of the metal changed accordingly. The objective of this work is to show that it is possible to obtain different optical responses of the crystal by introducing changes in certain structure's parameters, regardless of values of dielectric permittivities of metal or dielectric medium. This approach is justified because there are also other parameters that may influence the optical response of the structure, like the ratio between the field wavelength and the horizontal period of the grating, in addition to the filling factor or the layer thickness.

\section{Numerical evaluation of the far field}

In this section we analyse different optical responses of the crystal within the range of the far field above and below the structure. Reflection $(R)$, transmission $(T)$ and absorption $(A=1-R-T)$ curves versus the field wavelength are presented for two different layer thicknesses $d$ and filling factors $f$. Figures 3 and 4 show the optical responses of the structures with $f=0.2$ and 0.65 , respectively, for two thicknesses $d=60 \mathrm{~nm}$ and $d=200 \mathrm{~nm}$ and for the incident field of TM and TE polarization. For each set of geometrical parameters there are visible differences in reflection and absorption curves between the cases of TE and TM polarizations.

As we already pointed out, both thickness and filling factors qualitatively change the character of the structure response and are responsible for its various optical properties. As expected, for low $f$ and TM polarization the crystal is transmissive in a very wide wavelength range. On the other hand, for high $f$ and TE polarization it acts as the almost perfect reflector. However, for other configurations it can act either as a good broadband or narrowband transmitter, in dependence on the type of polarization incidence.

For the small layer thickness $(d=60 \mathrm{~nm})$, the low $f$-factor the crystal works as the transmissive structure in a wide range of wavelengths and for both, TM and TE polarizations. The increase of reflection from about $670 \mathrm{~nm}$ wavelength limits the high-transmission band at the long-wavelength side for incidence of TE polarization. This is in agreement with a cut-off wavelength for TE polarized wave propagating in a metal/dielectric/metal slit: $\lambda_{\text {co }}=2 \Lambda(1-f)=640 \mathrm{~nm}$ [16]. In the case of TM polarization, the structure hardly interacts with the incident wave and their reflection and absorption levels approaches nearly zero in the presented wavelength range.

With increasing thickness (to $d=200 \mathrm{~nm}$ ), the broad and low peak of increased reflection in a long-wavelength range can be observed for incidence of TM polarization. On the other hand, for TE polarization, the optical response of the crystal changes remarkably. In the range 
TM
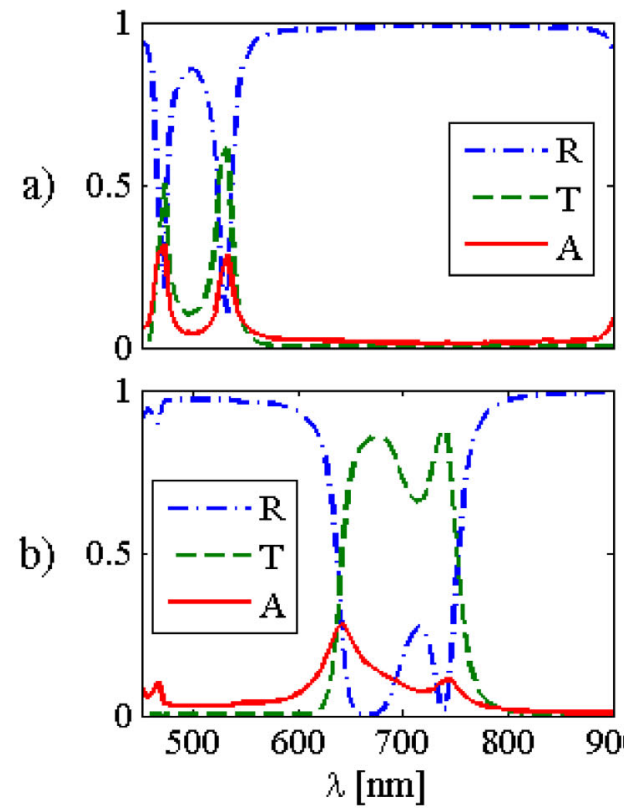

TE
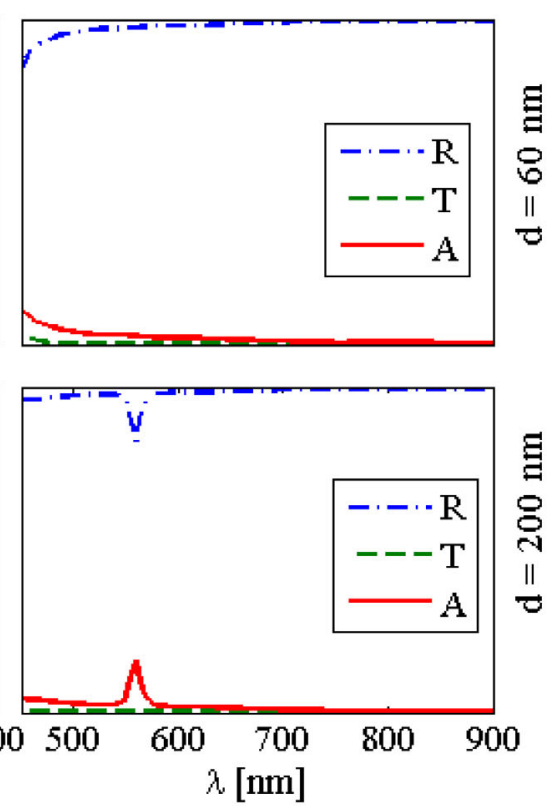

Fig. 4. Wavelength dependence of reflection $(R$ - blue dotted-dashed line), transmission ( $T$ - green dashed line) and absorption ( $A$ - red solid line) for the configurations with $f=0.65$. (a) The thickness $d=60 \mathrm{~nm}$ for incidence of TM (left) and TE (right) polarization; (b) the thickness $d=200 \mathrm{~nm}$ for incidence of TM (left) and TE (right) polarization.

550-700 $\mathrm{nm}$ in which high transmission can be observed in the thin crystal, the structure becomes now a very good reflector. Moreover, three narrow bands, corresponding to high transmission and increasing absorption, appear at longer wavelengths of the incident field.

Optical responses of the high $f$-factor crystals strongly depend on the polarization of the incident field too (see Fig. 4). They are, in general, still characterized by relatively low absorption. As expected, the crystal shows strong and broadband reflection for TE polarization. Moreover, it exhibits slightly different behaviour with increasing thickness for TM polarization due to the increase of the metal thickness and the increased separation of the layers. A double peak of narrowband transmission accompanied with absorption shifts spectrally to longer wavelengths as the thickness increases and transforms into a broad transmission band. Thus, manipulation of the layer thickness influences on the width and magnitude of transmission and the high $f$-factor crystal acts like a polarization-dependent filter.

Therefore, it is possible to obtain either a broadband transmitter or a broadband reflector for both TM and TE polarization of the incident wave at the same time. The broadband transmitter can be designed with use of low filling fraction and low thicknesses of the layers. The broadband reflector, on the other hand, is obtained for high filling factors and low thicknesses.

\section{Numerical evaluation of the near field}

In addition to the far field response, the near field distributions of particular resonances facilitate the interpretation of the nature of modes excited in the structure and other phenomena occurring in the far field, as presented in the previous section. In the structure, the stripes in all layers are placed without any horizontal spatial shift. This fact has two consequences. First, it creates the stacked metal/insulator/metal (MIM) cavities of the thickness $d$ between the layers. Second, it creates air areas through the total crystal thickness in vertical direction. Those features lead to a rich spectrum of different crystal responses.

Metal nanostripes can be treated as elementary dipole oscillators [17]. Under the incidence of TM polarization the single metal nanostripe shows a localized plasmon resonance in its optical response. This is a consequence of the collective oscillation of conduction band electrons in metal [17]. Since the metal stripes are separated by a distance shorter than the wavelength of the incident field, near field coupling dominates over the far field coupling. Interactions between the neighbouring stripes introduce spectral shifts of the localized resonance wavelengths. Since the skin depth of silver is between 22 and $27 \mathrm{~nm}$ in this wavelength range, the EM field coupling through the $60 \mathrm{~nm}$ (or more) thick metal stripes is weak. Thus, the localized plasmon modes are attributed mainly to the cavities between metal stripes, not to the stripes themselves. However, for $d=60 \mathrm{~nm}$ the coupling through the metal does exist as well.

\subsection{Crystals of low f-factor}

In the low- $f$ crystals two important phenomena appear visible - localized modes are excited and the transmission occurs in the free spaces between the metal ridges. 

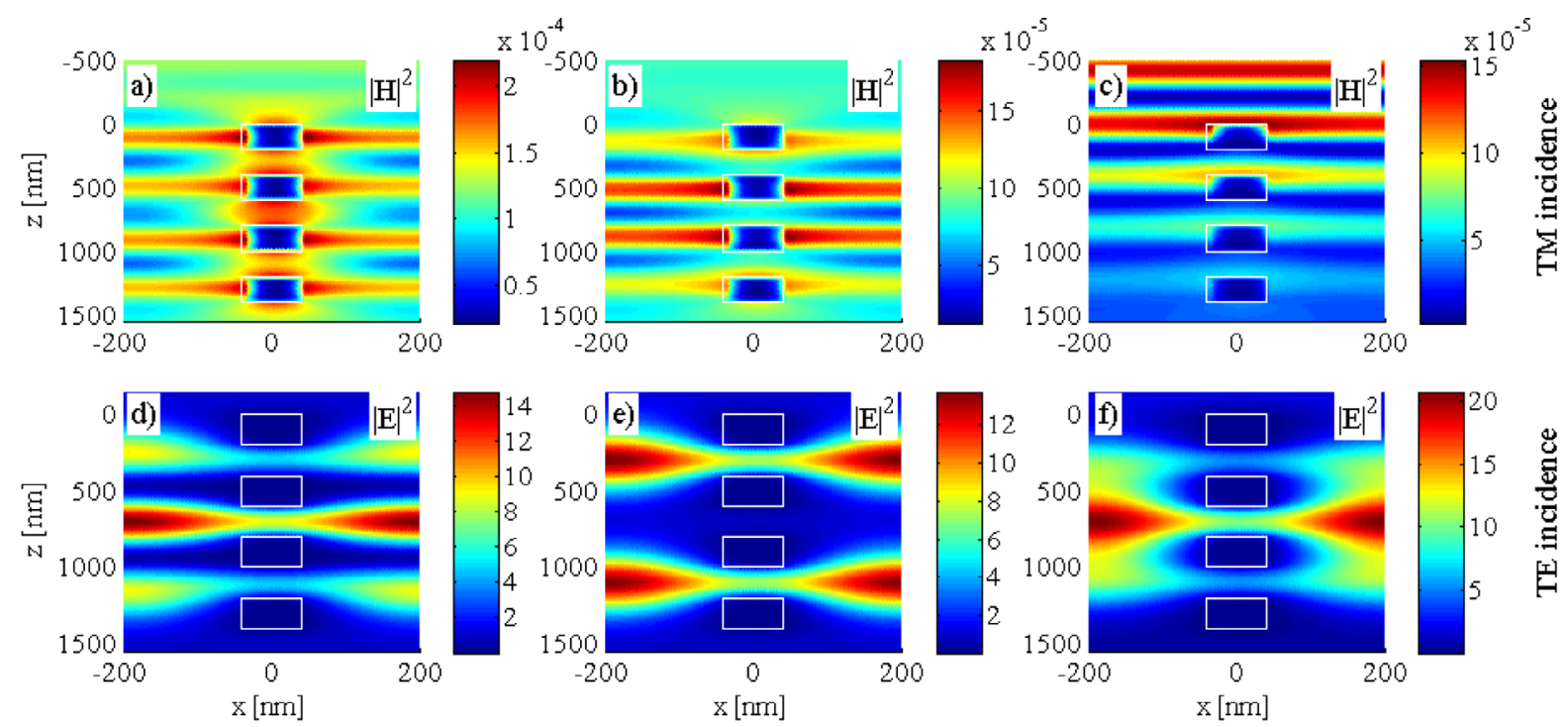

Fig. 5. Magnetic and electric field distributions for the configuration with $f=0.2$ and $d=200 \mathrm{~nm}$ : (a) $\lambda=580 \mathrm{~nm}$ (incidence of TM polarization), (b) $\lambda=680 \mathrm{~nm}$ (TM), (c) $\lambda=850 \mathrm{~nm}$ (TM), (d) $\lambda=725 \mathrm{~nm}$ (TE, 3rd order resonance), (e) $\lambda=812 \mathrm{~nm}$ (TE, 2nd order resonance), (f) $\lambda=873 \mathrm{~nm}$ (TE, 1st order resonance).

Moreover, for closely spaced metal stripes $(d<\lambda)$, interactions between the local EM field near the metal surface dominates [18].

For TM polarization the structure behaves as a periodic set of vertical metallic nanostripe chains [9]. The magnetic near field corresponding to the high transmission region is presented in Figures $5 \mathrm{a}$ and $5 \mathrm{~b}$. The localized plasmon modes excited at the individual metal stripes are coupled through neighbouring stripes. At shorter wavelengths both transverse and longitudinal modes are present. Vertical and horizontal coupling between neighbouring stripes is visible, especially in Figure 5a. The energy is transported along the chain by field coupling between the localized modes of neighbouring stripes [19]. Those modes are the gap surface plasmons in MIM cavities between every second metal stripe [20]. The gap plasmons are excited at the surfaces of the upper and lower metal stripes and can be coupled through the dielectric space between stripes.

In the area of reduced transmission $(\lambda \approx 750-950 \mathrm{~nm})$ (Fig. 5c) there is no vertical coupling between modes. At longer wavelengths the transverse mode perpendicular to the chain dominates. In [9] the authors indicate that for short nanostripe chains the dominating collective mode is a longitudinal one, however the analysed structure configurations differ significantly in this respect, as they analysed the single chain of wires of circular cross section separated by $5 \mathrm{~nm}$. All those differences may cause changes in the optical response. In the case considered here there is no single resonant transmission. Instead, the broad transmission band persists.

Figures $5 \mathrm{~d}-5 \mathrm{f}$ present electric field corresponding to three peaks in Figure 3 for $d=200 \mathrm{~nm}$ and the incidence of TE polarization. The field distribution corresponds to first (Fig. 5f), second (Fig. 5e) and third (Fig. 5d) resonance. The order of the resonances is determined by the phase $\pi$ changes of the field in the direction perpendicular to the layers.

For TE polarization three transmission resonances are the collective Fabry-Perot resonances of different orders. These resonances are excited between the gratings in the structure [21] and are shifted spectrally to longer wavelengths with increasing thickness of $d$ (the transmission maximum for configuration with $d=60 \mathrm{~nm}$ indicates in fact the first Fabry-Perot resonance). The cavity modes accumulate energy in the near field, leading to enhanced absorption (10-15\%), apparent as a high field intensity inside crystal structure [14].

\subsection{Crystals of high f-factor}

Structures with $f=0.65$ show strong dependence on polarization of the incident field. For TE polarization they are characterized by a high, broadband reflection. For such the high $f$-factor the cross-section of the vertical waveguide is too small to transmit TE polarized wave. For TM polarization there are transmission peaks, which are shifted spectrally to longer wavelengths with the increasing thickness. In Figure 6 we present the magnetic field distributions for TM polarization corresponding to different incidence wavelengths. For thin structures $(d=60 \mathrm{~nm})$ there is a field coupling through the metal stripes and a collective mode is excited in the structure. The first and second Fabry-Perot vertical resonances correspond to two transmission peaks. Additionally, the horizontal coupling between neighbouring vertical waveguides is also clearly visible.

Here the strong influence of the number of layers on the structure's modes and optical response is obvious. With increasing number of layers the wavelength of the collective modes in the structure would shift spectrally to longer 


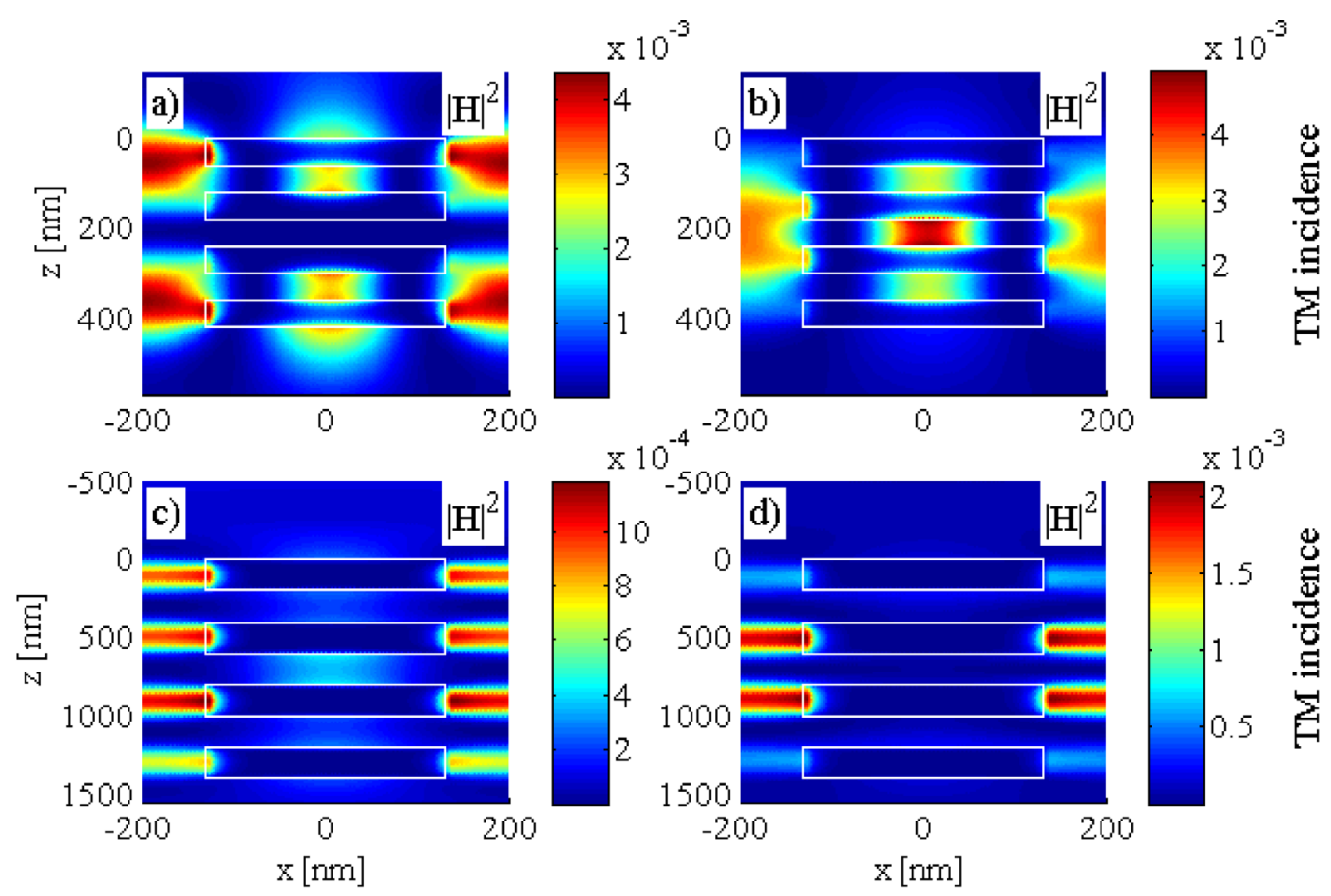

Fig. 6. Magnetic field distributions for the configuration with $f=0.65$ and incidence of TM polarization: (a) $d=60 \mathrm{~nm}$, $\lambda=471 \mathrm{~nm}$, (b) $d=60 \mathrm{~nm}, \lambda=530 \mathrm{~nm}$, (c) $d=200 \mathrm{~nm}, \lambda=675 \mathrm{~nm}$, (d) $d=200 \mathrm{~nm}, \lambda=738 \mathrm{~nm}$.
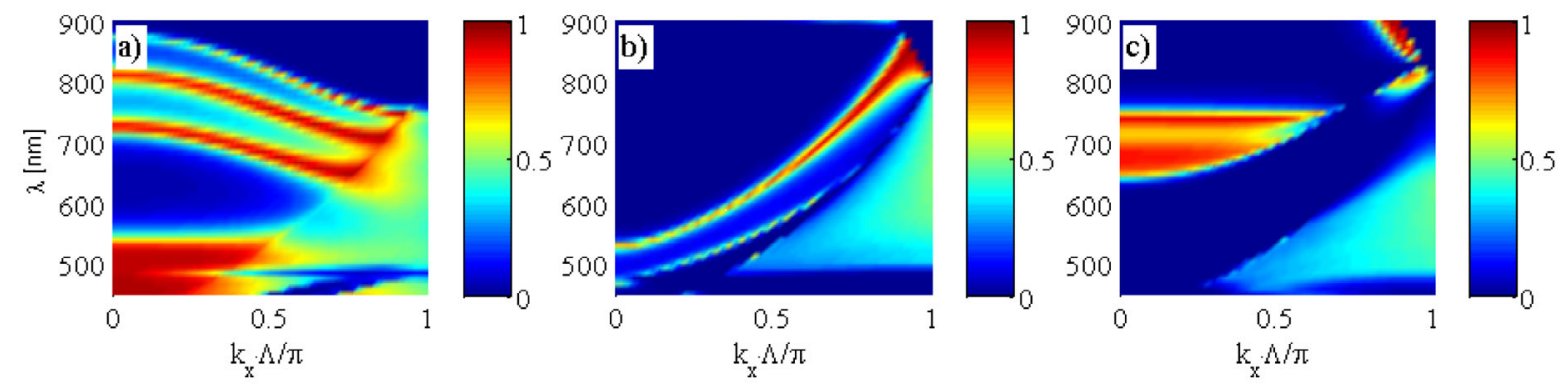

Fig. 7. Dispersion relation (dependence of transmission on the incident wavelength and wave vector) for the configurations with (a) $f=0.2, d=200 \mathrm{~nm}$ and TE polarization, (b) $f=0.65, d=60 \mathrm{~nm}$ and TM polarization, $(\mathrm{c}) f=0.65, d=200 \mathrm{~nm}$ and TM polarization.

wavelengths. This is not the case for thicker structures, where coupling through the metal becomes negligible. Here the main coupling occurs horizontally between neighbouring vertical chain waveguides (Figs. 6c and 6d). Note that the TM wave does not possesses a waveguide cut-off and can pass directly through the structure.

\section{Band structure analysis}

To conclude our analysis of square lattice crystals, in Figure 7 we present the dispersion relations for low- $f$ and high- $f$ structures along the incidence $k_{x}$ component for different thicknesses and polarizations. The dispersive modes exist in each type of crystal. However, three resonance Fabry-Perot modes corresponding to $f=0.2$, $d=200 \mathrm{~nm}$ and TE polarization (Fig. 7a) shift to shorter wavelengths at higher incidence angles, in opposite to resonance modes corresponding to $f=0.65, d=60 \mathrm{~nm}$ and TM polarization, which redshift. This is a consequence of different mechanisms of mode excitation at different wave polarization incidence. On the other hand, localized plasmons coupled through gaps between neighbouring vertical wire chains (Fig. 7c) show no dispersion, which is in agreement with conclusions in $[22,23]$.

\section{Crystals with spatially shifted layers}

To compare the optical properties of the configuration without a horizontal shift between the layers with a more complicated, spatially shifted structure, we analyse in this section the 4-layer crystal with a spatial shift by half of the horizontal spacing introduced in every second grating layer (Fig. 1b). Thus, this structure consists of the two 
$\mathrm{TM}$
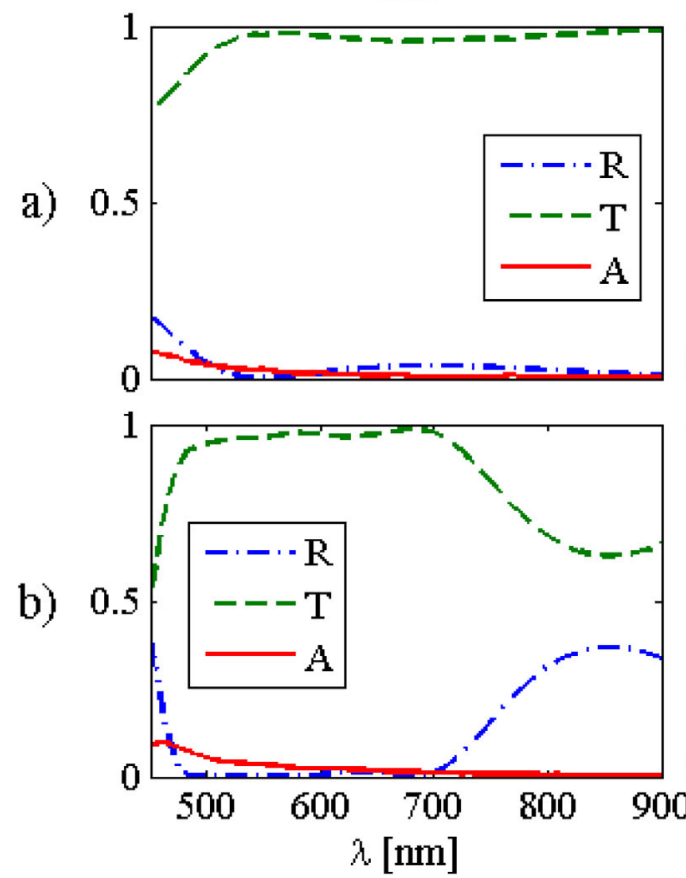

TE
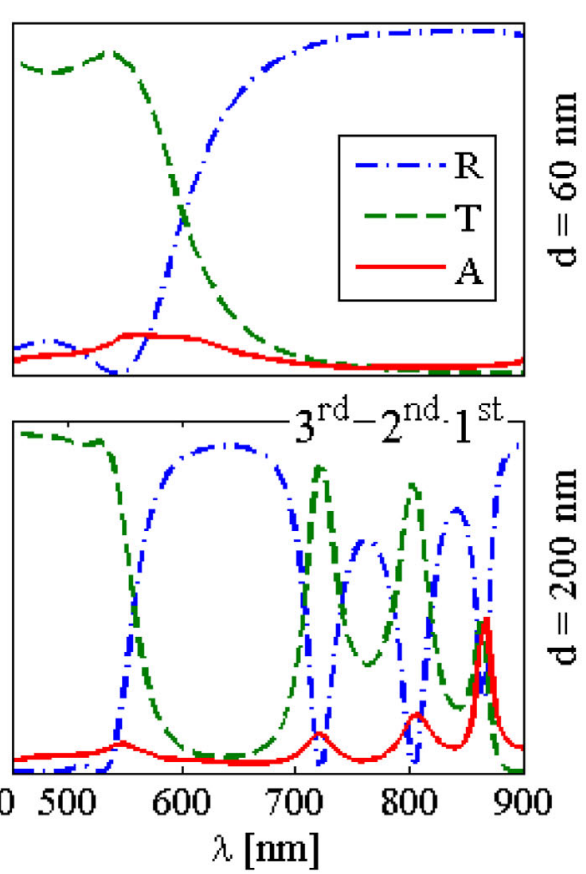

Fig. 8. Wavelength dependence of reflection $(R-$ blue dotted-dashed line), transmission ( $T$ - green dashed line) and absorption ( $A$ - red solid line) for 4-layer crystal with spatial shift for the configurations with $f=0.2$. (a) The thickness $d=60$ nm for incidence of TM (left) and TE (right) polarization; (b) the thickness $d=200 \mathrm{~nm}$ for incidence of TM (left) and TE (right) polarization. The resonances of the structure: 1 st order resonance at $\lambda=865$ nm, 2nd order resonance at $\lambda=803$ nm and 3 rd order resonance at $\lambda=722 \mathrm{~nm}$ are also indicated.

2-layer structure periodic in the (vertical) propagation direction. In Figures 8 and 9 we present the reflection, transmission and absorption curves in dependence on the incident wavelength for the low and high filling factors and for the same other geometrical parameters as in Figures 3 and 4 . It should be noted, that the horizontal spatial shift between two layers presented in [24] was introduced in order to modify the horizontal propagation direction of surface plasmon polaritons in the near field of the structure. However, in the case considered here, this shift is symmetric with respect to the horizontal $x$-direction. Therefore, the horizontal spatial shift does not change significantly the optical response of the crystal in the range of far field, in most configurations discussed here.

Optical responses for TE incidence remain qualitatively the same as in the case of the structure without the horizontally symmetric shift between the layers, except the narrowed transmission band for thin metal structure $(d=60 \mathrm{~nm})$ and low filling factor $(f=0.2)$. In this case the structure can no longer be considered as a MIM slit cavity. The cut-off frequency is then shifted spectrally to shorter wavelengths due to additional disturbance introduced by the spatially shifted layers. On the other hand, for low filling factors and large height $d$ of the layers, the horizontal shift of them does not influence much on the optical response of the crystal.

In contrast, the broadening of the transmission band for $d=200 \mathrm{~nm}$ and significant spectral shift to longer wavelengths of transmission peaks for $d=60 \mathrm{~nm}$ are the important changes in the spectra for the structure with $f=0.65$ under incidence of TM polarized wave. Excitation conditions of the collective modes in the spatially shifted structure are changed. Now the cavities between layers are vertically extended due to the spatial shift of the every second layer. This is the cause of significant spectral shift to longer wavelengths of the Fabry-Perot modes for $d=60 \mathrm{~nm}$ and TM polarization.

Figure 10 presents the field distributions for the particular resonances presented in Figures 8 and 9. Figures 10a-10c display third-, second- and first-order resonance, respectively. The position change of subsequent field maxima is clearly visible. Horizontally continuous mode field distribution of the spatially shifted structure (Figs. 10a-10c) corresponds to the almost horizontally discontinuous field distribution presented in Figures 5d-5f. Figures $10 \mathrm{~d}$ and $10 \mathrm{e}$ show two transmission maxima originating from the collective modes in the structure. Extended gaps between metal stripes in vertical direction cause the spectral shift to longer wavelengths of the resonances and changes in the field distribution. Figure $10 \mathrm{f}$ presents the magnetic field distribution corresponding to high transmission without a direct line of sight through the crystal. Fields are similar to those obtained for the structure without a horizontal shift between the layers despite the fact, that upper metal stripes screen the air gaps in lower grating layers. This feature is in agreement with results presented in [25], where a bilayer structure with introduced spatial shift of a half of grating period 
TM

a)
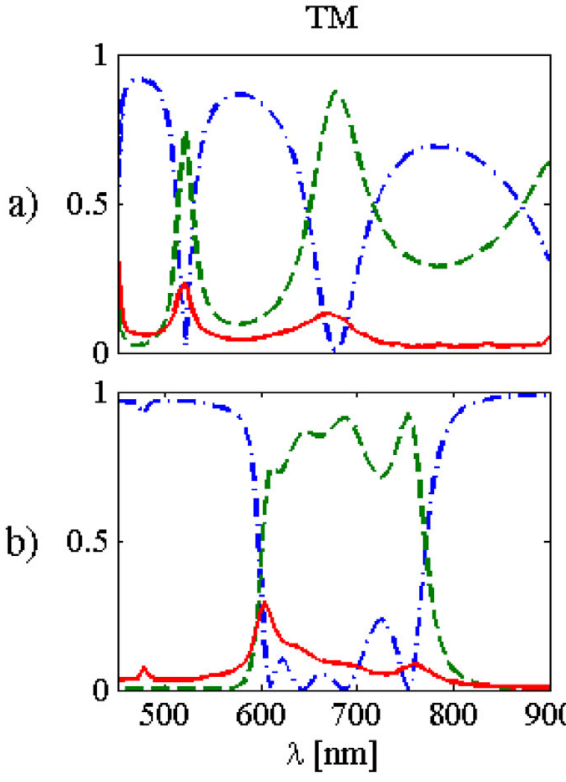

TE
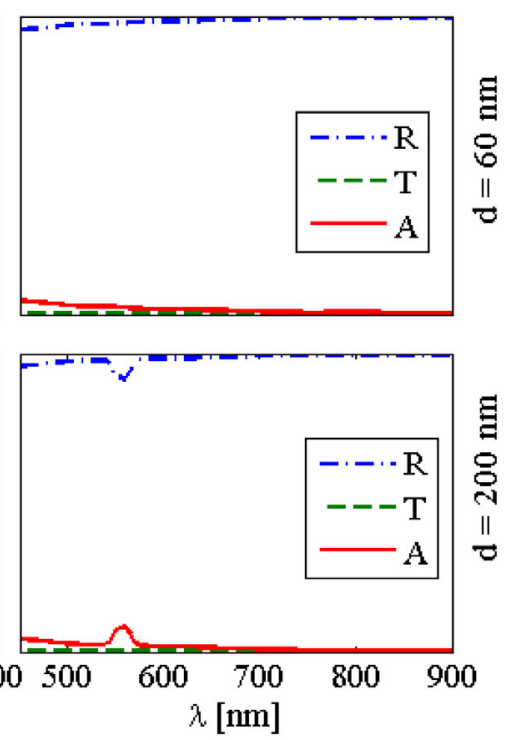

Fig. 9. Wavelength dependence of reflection $(R$ - blue dotted-dashed line), transmission ( $T$ - green dashed line) and absorption ( $A$ - red solid line) for 4-layer crystal with spatial shift for the configurations with $f=0.65$. (a) Thickness $d=60 \mathrm{~nm}$ for incidence of TM (left) and TE (right) polarization; (b) thickness $d=200 \mathrm{~nm}$ for incidence of TM (left) and TE (right) polarization.

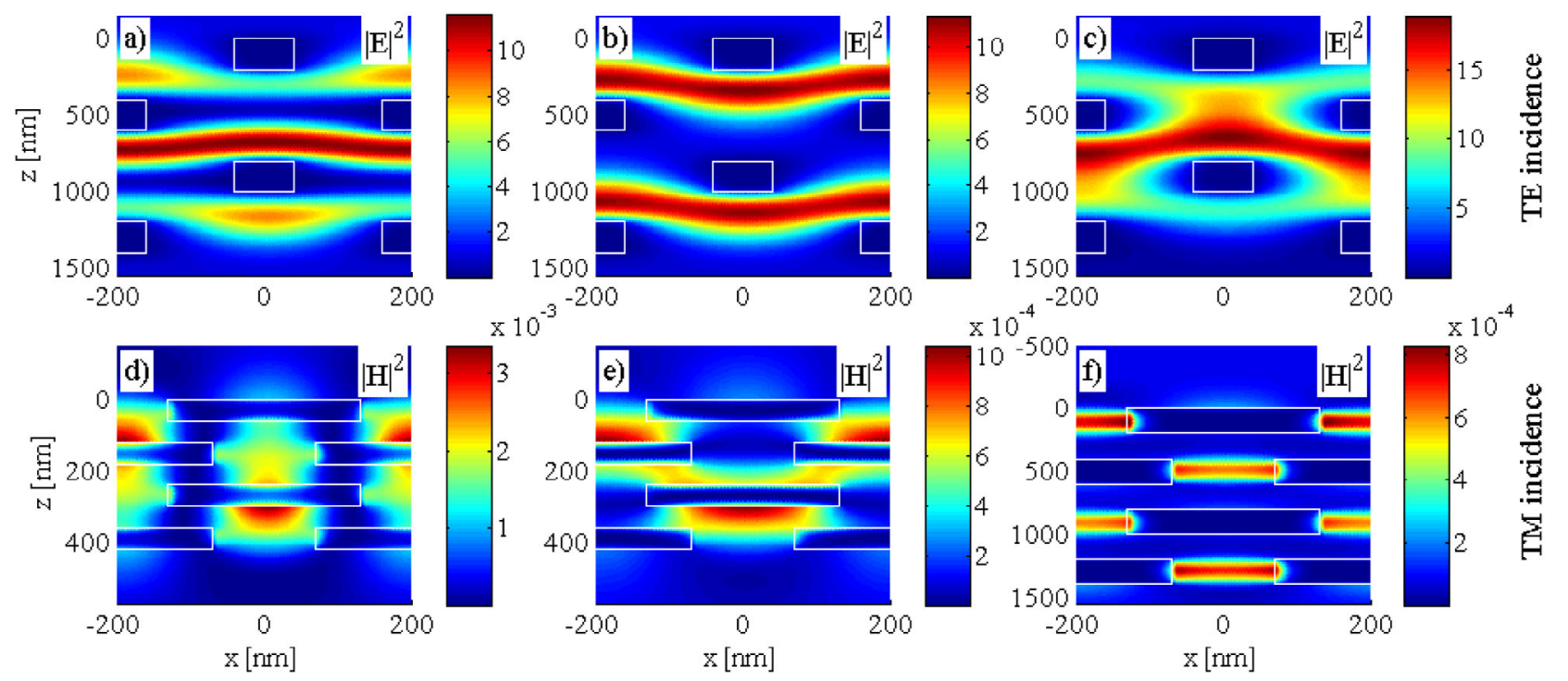

Fig. 10. Electric and magnetic field distributions: (a) $d=200 \mathrm{~nm}, \lambda=722 \mathrm{~nm}$ (TE, 3rd order resonance), (b) $d=200 \mathrm{~nm}$, $\lambda=803 \mathrm{~nm}$ (TE, 2nd order resonance), (c) $d=200 \mathrm{~nm}, \lambda=865 \mathrm{~nm}$ (TE, 1st order resonance), (d) $d=60 \mathrm{~nm}, \lambda=521$ $\mathrm{nm}(\mathrm{TM}),(\mathrm{e}) d=60 \mathrm{~nm}, \lambda=678 \mathrm{~nm}$ (TM), (f) $d=60 \mathrm{~nm}, \lambda=686 \mathrm{~nm}(\mathrm{TM})$. Figures a-c are analogous to Figures $5 \mathrm{~d}-5 \mathrm{f}$. Figures $\mathrm{d}$ and e are analogous to Figures $6 \mathrm{a}$ and $6 \mathrm{~b}$. Figure $\mathrm{f}$ is analogous to Figures $6 \mathrm{c}$ and $6 \mathrm{~d}$.

showed high resonant transmission despite the lack of direct line of sight. However, the analysed structure shows significantly broader transmission band.

Finally, the question arises whether the transmission and absorption characteristics of the structure discussed in this paper can be further optimized. It seems that it may be accomplished, for example, by changes of the dielectric separation between the periodic stripe layers or by changes of the period value of the horizontal gratings. It might be also reasonable to expect new effects, for exam- ple, in the 3D crossed structure (both with or without the horizontal spatial shift), where every second grating layer is in addition rotated horizontally by $\pi / 2$.

Note that our numerical simulations are performed for the relatively simple field-structure configuration. The structure is horizontally periodic in the direction parallel to the incidence plane and incidence of the plane wave of the linear - TM or TE - polarization is normal with respect to the horizontal surface of the structure. In this case the standard TM and TE Fresnel coefficients 
TM

a)
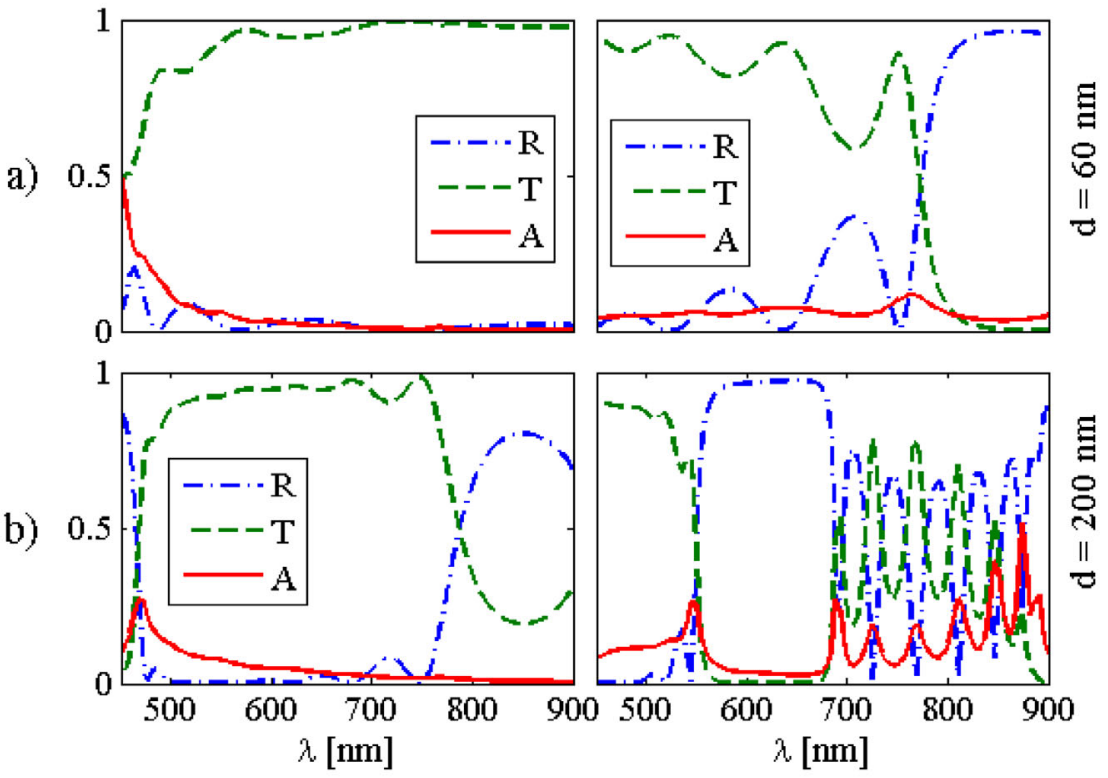

Fig. 11. Eight-layer crystal with rectangular lattice. Wavelength dependence of reflection $(R-$ blue dotted-dashed line), transmission ( $T$ - green dashed line) and absorption $(A$ - red solid line) for the configurations with filling factor $f=0.2$. (a) The thickness $d=60 \mathrm{~nm}$ for incidence of TM (left) and TE (right) polarization; (b) the thickness $d=200 \mathrm{~nm}$ for incidence for TM (left) and TE (right) polarization.

of transmission and reflection are suitable for the field evaluation in its separate polarization components.

Note also that in more general three-dimensional (3D) field-structure configurations, where, for example, the structure is horizontally periodic in two mutually orthogonal directions or incidence of the plane wave of arbitrary polarization is oblique, the cross-polarization coupling (XPC) between the TM and TE field components come into play and modify the field transmission and reflection coefficients. However, considerations on such complex 3D field-structure configurations are beyond the scope of this work. Examples of them were already analysed by us in the context of interactions of paraxial beams with planar isotropic interfaces [26], open nano-meta-rezonators [27] and periodic arrays of Fresnel zone plates [28].

\section{Crystals with more layers}

In this section we compare action of the photonic crystals of square and triangular lattices consisting of four layers presented in previous sections with analogical crystals consisting of eight layers. Figures 11-14 present reflection, transmission and absorption spectra, analogous to Figures 3, 4, 8 and 9, respectively. The spectra are qualitatively very similar to the spectra of four-layer crystals. The main difference is the number of resonance peaks visible in particular spectra. With increased number of layers the number of transmission maxima (reflection minima) in certain cases increases. These changes are understandable when we analyse closer the character of the resonances.
For rectangular lattice crystal with $f=0.2$ and TE polarization the transmission resonances origin from the collective Fabry-Perot modes excited between the gratings. Additional crystal layers form larger number of cavities in which those resonances are excited, producing additional resonance peaks.

Similarly, for rectangular lattice crystal with $f=0.65$, $d=60 \mathrm{~nm}$ and TM polarization the field coupling through the metal stripes and a collective mode in the structure are responsible for transmission peaks. Additionally, for $d=200 \mathrm{~nm}$ the horizontal coupling between neighbouring vertical waveguides also results in larger number of resonance peaks when we double the number of layers.

Similarly, in the case of triangular lattice crystals, increasing number of layers also results in larger number of resonance peaks. However, other features of the eightlayer crystal action remain much the same as in the case of the four-layer crystals. Therefore, the proper choice of geometrical and field parameters makes the action of the four-layer structure similar to the action of a semiinfinite crystal of both rectangular or triangular lattice cases. Near-total transmission can be obtained for TM field polarization and $f=0.2$ as shown in Figures 11 and 13. Similarly, the case of almost total reflection is reached for TE field polarization and $f=0.65$, as presented in Figures 12 and 14.

For a specified TM or TE field polarization and a certain range of values of the filling factor the results obtained for a few crystal layers can be regarded as the proper approximation for the case of a semi-infinite crystal structure. 
TM

a)
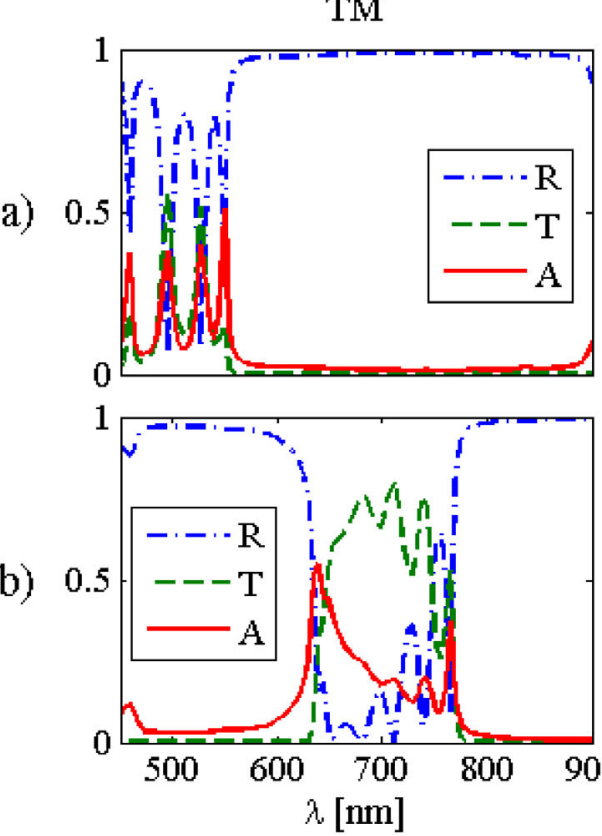

TE
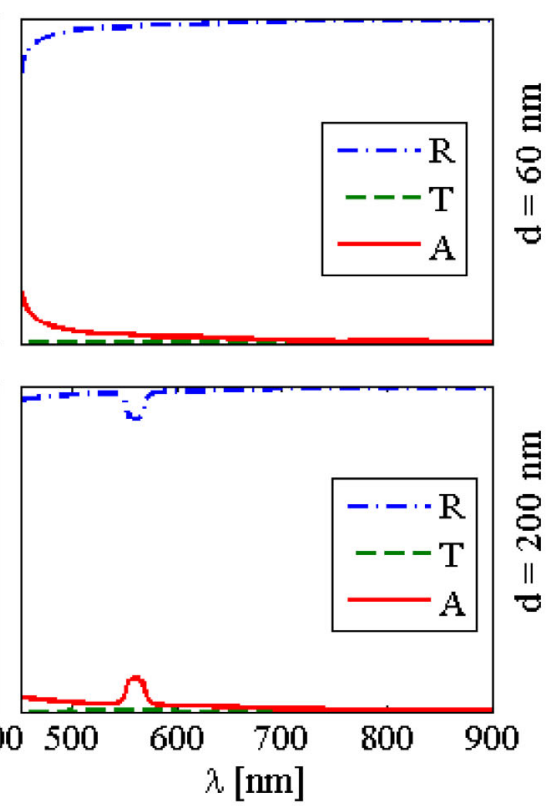

Fig. 12. Eight-layer crystal with rectangular lattice. Wavelength dependence of reflection $(R-$ blue dotted-dashed line), transmission ( $T$ - green dashed line) and absorption $(A$ - red solid line) for the configurations with $f=0.65$. (a) The thickness $d=60 \mathrm{~nm}$ for incidence of TM (left) and TE (right) polarization; (b) the thickness $d=200 \mathrm{~nm}$ for incidence of TM (left) and TE (right) polarization.

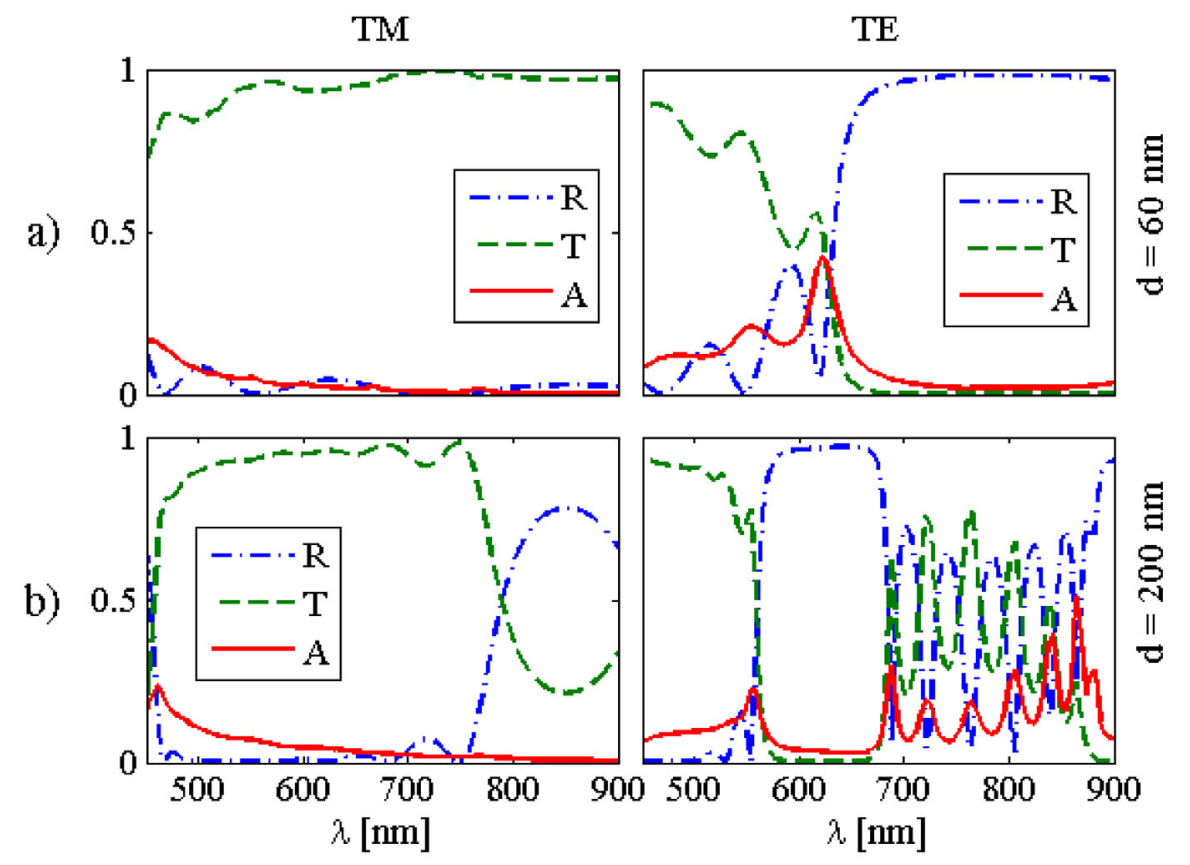

Fig. 13. Eight-layer crystal with triangular lattice. Wavelength dependence of reflection ( $R$ - blue dotted-dashed line), transmission $(T-$ green dashed line) and absorption $(A-$ red solid line) for the configurations with filling factor $f=0.2$. (a) The thickness $d=60 \mathrm{~nm}$ for incidence of TM (left) and TE (right) polarization; (b) the thickness $d=200 \mathrm{~nm}$ for incidence for TM (left) and TE (right) polarization. 
TM

a)

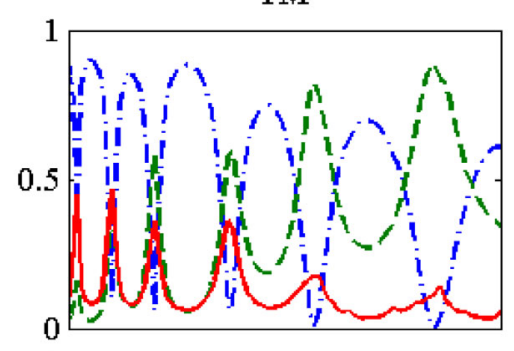

b)

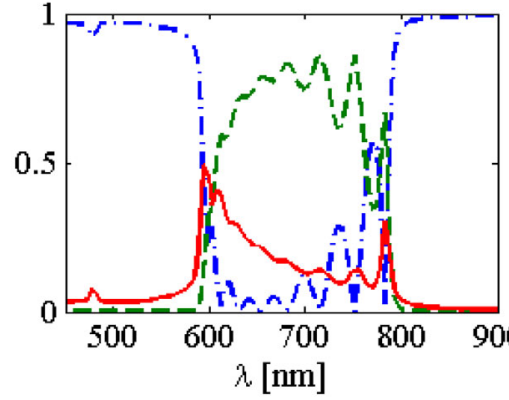

TE

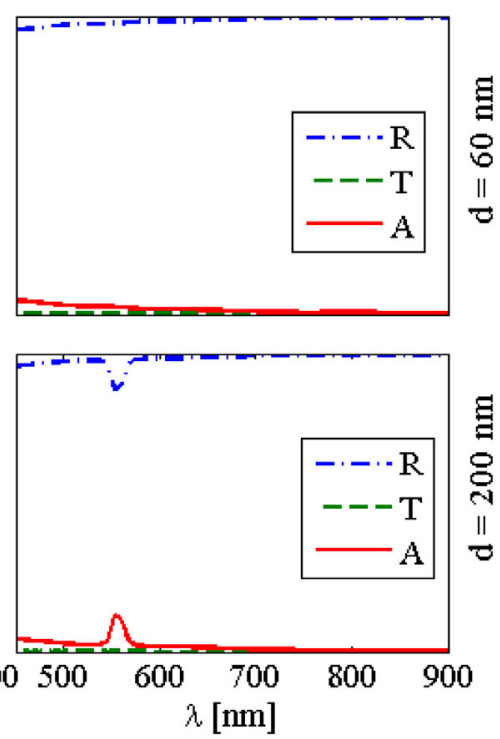

Fig. 14. Eight-layer crystal with triangular lattice. Wavelength dependence of reflection ( $R$ - blue dotted-dashed line), transmission ( $T$ - green dashed line) and absorption $(A-$ red solid line) for the configurations with $f=0.65$. (a) The thickness $d=60 \mathrm{~nm}$ for incidence of TM (left) and TE (right) polarization; (b) the thickness $d=200 \mathrm{~nm}$ for incidence of TM (left) and TE (right) polarization.

\section{Conclusions}

In summary, we have numerically shown that the silver stripe crystals can exhibit various optical properties with respect to specified changes of its geometrical parameters. Qualitative differences in the properties of the structure depend mainly on the thickness and filling factor of each layer. In both cases (with and without the horizontal spatial shift), the optical response of the crystal is characterized by small absorption. For structures with thin stripes $(d=60 \mathrm{~nm})$, the collective modes are responsible for high transmission. For thicker structures $(d=200 \mathrm{~nm})$ the main role in modifying of the spectral response plays the near field coupling between localized resonances at particular stripes. Various optical properties of the metal stripe $2 \mathrm{D}$ crystal is demonstrated.

In dependence on its geometrical parameters and polarization of incidence waves it can work as:

- a broadband reflector (for $f=0.65$ and TE polarization with or without a spatial shift and for $f=0.65$, $d=60 \mathrm{~nm}$ and TM polarization without a spatial shift),

- a broadband transmitter (for $f=0.2$ and TM polarization, for $f=0.2, d=60 \mathrm{~nm}$ and TE polarization and for $f=0.65, d=200 \mathrm{~nm}$ and TM polarization; in all these cases with or without a spatial shift),

- a narrowband transmitter (for $f=0.2, d=200 \mathrm{~nm}$ and TE polarization or for $f=0.65, d=60 \mathrm{~nm}$ and TM polarization in both these cases with or without a spatial shift).

We also showed that the horizontally symmetric spatial shift of every second grating layer by a half of the period of the horizontal lattice does not change qualitatively the optical response in the most structure configurations considered in this paper.

This work was partially supported by the National Science Centre, Poland, under Grant No. 2015/19/B/ST7/03656.

\section{Author contribution statement}

A.R. simulations, W.N. synthesis.

Open Access This is an open access article distributed under the terms of the Creative Commons Attribution License (http://creativecommons.org/licenses/by/4.0), which permits unrestricted use, distribution, and reproduction in any medium, provided the original work is properly cited.

\section{References}

1. J.D. Joannopoulos, S.G. Johnson, J.N. Winn, R.D. Meade, photonic crystals: molding the flow of light, 2nd ed. (Princeton University Press, Princeton, Oxford, 2008)

2. S.A. Maier, Plasmonics: fundamentals and applications (Springer, New York, 2007)

3. Z. Sun, X. Zuo, J. Li, Plasmonics 6, 745 (2011)

4. S.G. Babiker, S. Yong, M.O. Sid-Ahmed, X. Ming, J. Electron. Cooling Therm. Control 4, 39 (2014)

5. J. Yu, Y. Shen, X. Li, R. Fu, J. Zi, Z. Zhu, J. Phys.: Condens. Matter 16, L51 (2004)

6. K. Kempa, A. Rose, Bull. Pol. Acad. Sci. Tech. Sci. 57, 35 (2009) 
7. J.N. Winn, Y. Fink, S. Fan, J.D. Joannopoulos, Opt. Lett. 23, 1573 (1998)

8. Y. Zeng, X. Chen, W. Lu, Physica E 30, (2005)

9. H.S. Chu, W.B. Ewe, W.S. Koh, E.P. Li, Appl. Phys. Lett. 92, 103103 (2008)

10. M.G. Moharam, E.B. Grann, D.A. Pommet, T.K. Gaylord, J. Opt. Soc. Am. A 12, 1068 (1995)

11. L. Li, J. Opt. Soc. Am. A 13, 1024 (1996)

12. L. Li, J. Opt. Soc. Am. A 13, 1870 (1996)

13. P.B. Johnson, R.W. Christy, Phys. Rev. B 6, 4370 (1972)

14. A. Roszkiewicz, W. Nasalski, J. Phys. B: At. Mol. Opt. Phys. 46, 025401 (2013)

15. M.G. Moharam, T.K. Gaylord, J. Opt. Soc. Am. 73, 1105 (1983)

16. N. Marcuvitz, Waveguide handbook (Dover Publications, Inc., New York, 1965)

17. A. Christ, T. Zentgraf, S.G. Tikhodeev, N.A. Gippius, O.J.F. Martin, J. Kuhl, H. Giessen, Phys. Status Solidi B 243, 2344 (2006)

18. S.A. Maier, P.G. Kik, H.A. Atwater, Phys. Rev. B 67, 205402 (2003)
19. J. Du, S. Liu, Z. Lin, J. Zi, S.T. Chui, Phys. Rev. A 79, 051801 (2009)

20. J. Jung, T. Søndergaard, S.I. Bozhevolnyi, Phys. Rev. B 79, 035401 (2009)

21. M. Chen, S.-Y. Lin, H.-C. Chang, A.S.P. Chang, Phys. Rev. B 78, 085110 (2008)

22. A. Christ, T. Zentgraf, S.G. Tikhodeev, N.A. Gippius, J. Kuhl, H. Giessen, Phys. Rev. B 74, 155435 (2006)

23. R. Ameling, D. Dregely, H. Giessen, Opt. Lett. 36, 2218 (2011)

24. A. Roszkiewicz, W. Nasalski, J. Phys. B: At. Mol. Opt. Phys. 43, 185401 (2010)

25. H.B. Chan, Z. Marcet, K. Woo, D.B. Tanner, D.W. Carr, J.E. Bower, R.A. Cirelli, E. Ferry, F. Klemens, J. Miner, C.S. Pai, J.A. Taylor, Opt. Lett. 31, 516 (2006)

26. W. Nasalski, Phys. Rev. E 74, 056613 (2006)

27. W. Szabelak, W. Nasalski, J. Phys. B: At. Mol. Opt. Phys. 44, 215403 (2011)

28. A. Roszkiewicz, W. Nasalski, J. Phys. B: At. Mol. Opt. Phys. 47, 165401 (2014) 